\title{
Smart Grid Technologies: Communication Technologies and Standards
}

\author{
Vehbi C. Gungor, Dilan Sahin, Taskin Kocak, Salih Ergüt, Concettina Buccella, \\ Carlo Cecati, Gerhard P. Hancke
}

\begin{abstract}
For a hundred years, there has been no change in the basic structure of the electrical power grid. Experiences have shown that the hierarchical, centrally-controlled grid of the twentieth century is ill-suited to the needs of the twenty-first. To address the challenges of the existing power grid, the new concept of smart grid has emerged. The smart grid can be considered as a modern electric power grid infrastructure for enhanced efficiency and reliability through automated control, high power converters, modern communications infrastructure, sensing and metering technologies, and modern energy management techniques based on the optimization of demand, energy and network availability, and so on. While current power systems are based on a solid information and communication infrastructure, the new smart grid needs a different and much more complex one, as its dimension is much larger. This paper addresses critical issues on smart grid technologies primarily in terms of information and communication technology (ICT) issues and opportunities. The main objective of this paper is to provide a contemporary look at the current state of the art in smart grid communications as well as to discuss the still-open research issues in this field. It is expected that this paper will provide a better understanding of the technologies, potential advantages and research challenges of the smart grid and provoke interest among the research community to further explore this promising research area.
\end{abstract}

Index Terms-Smart Grid, Communication Technologies, Advanced Metering Infrastructure (AMI), Quality of Service (QoS), Standards.

\section{INTRODUCTION}

Today's electrical infrastructure has remained unchanged for about a hundred years. The components of the hierarchical grid are near to the end of their lives. While the electrical grid has been ageing, the demand for electricity has gradually increased. According to the U.S. Department of Energy report, the demand and consumption for electricity in the U.S. have increased by $2.5 \%$ annually over the last twenty years [1]. Today's electric power distribution network is very complex and ill-suited to the needs of the twenty-first century. Among the deficiencies are a lack of automated analysis, poor visibility, mechanical switches causing slow response times,

Vehbi C. Gungor, Dilan Şahin and Taskin Koçak, are with the Bahçeşehir University, Dept. of Computer Engineering, İstanbul, Turkey. e-mail: cagri.gungor, dilan.sahin, taskin.kocak@bahcesehir.edu.tr

Salih Ergüt is with Türk Telekom Group R\&D Division, İstanbul, Turkey. e-mail: salih.ergut@ turktelekom.com.tr

Concettına Buccella and Carlo Cecati are with the University of L'Aquila, Department of Industrial and Information Engineering and Economics, and with DigiPower Ltd. L'Aquila, Italy e-mail: concettina.buccella, carlo.cecati@univaq.it

Gerhard P. Hancke is with the University of Pretoria, Department of Electrical, Electronic and Computer Engineering, Pretoria, South Africa. email: g.hancke@ieee.org lack of situational awareness, etc. [2]. These have contributed to the blackouts happening over the past 40 years. Some additional inhibiting factors are the growing population and demand for energy, the global climate change, equipment failures, energy storage problems, the capacity limitations of electricity generation, one-way communication, decrease in fossil fuels and resilience problems [5]. Also, the greenhouse gas emissions on Earth have been a significant threat that is caused by the electricity and transportation industries [6]. Consequently, a new grid infrastructure is urgently needed to address these challenges.

To realize these capabilities, a new concept of next generation electric power system, the smart grid, has emerged. The smart grid is a modern electric power grid infrastructure for improved efficiency, reliability and safety, with smooth integration of renewable and alternative energy sources, through automated control and modern communications technologies [1], [11]. Renewable energy generators seem as a promising technology to reduce fuel consumption and greenhouse gas emissions [7]. Importantly, smart grid enabling new network management strategies provide their effective grid integration in Distributed Generation (DG) for Demand Side Management and energy storage for DG load balancing, etc. [8], [9]. Renewable energy sources (RES) are widely studied by many researchers [10] and the integration of RES, reducing system losses and increasing the reliability, efficiency and security of electricity supply to customers are some of the advances that smart grid system will increase [12]. The existing grid is lack of communication capabilities, while a smart power grid infrastructure is full of enhanced sensing and advanced communication and computing abilities as illustrated in Figure 1. Different components of the system are linked together with communication paths and sensor nodes to provide interoperability between them ,e.g., distribution, transmission and other substations, such as residential, commercial and industrial sites.

In the smart grid, reliable and real-time information becomes the key factor for reliable delivery of power from the generating units to the end-users. The impact of equipment failures, capacity constraints, and natural accidents and catastrophes, which cause power disturbances and outages, can be largely avoided by online power system condition monitoring, diagnostics and protection [1]. To this end, the intelligent monitoring and control enabled by modern information and communication technologies have become essential to realize the envisioned smart grid [1], [14].

USA, Canada, China, South Korea, Australia and European 


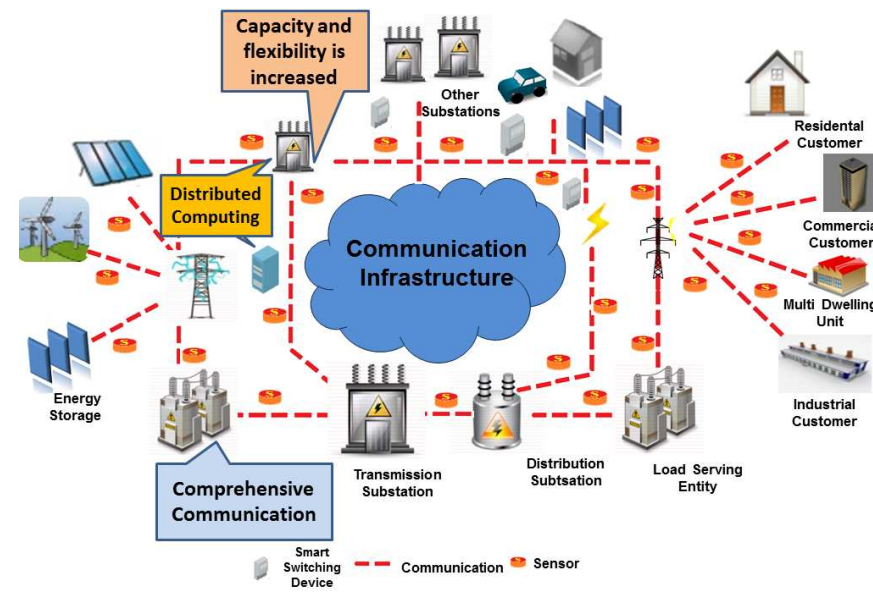

Figure 1. Smart grid architecture increases the capacity and flexibility of the network and provides advanced sensing and control through modern communications technologies.

Community (EC) countries has started doing research and development on smart grid applications and technologies. For example, the U.S. Government has announced the largest power grid modernization investment in the U.S. history, i.e., \$ 3.4 billion in grant awards, funding a broad range of smart grid technologies [2]. Local Distribution Companies (LDCs) are integrating advanced metering and two-way communication, automation technologies to their distribution systems [15]. In addition to research and development projects, many electric utilities are also taking incremental steps to make the smart grid technology a reality. Most of them are signing agreements with telecom operators or smart meter vendors to carry out smart grid projects. All these agreements define the main requirements and features of the necessary communications infrastructure to provide online communication between smart meters and the utility's back-haul system, i.e., the so-called advanced metering infrastructure (AMI). In general, the AMI is a two-way communications network and is the integration of advanced sensors; smart meters, monitoring systems, computer hardware, software and data management systems that enable the collection and distribution of information between meters and utilities [14].

In this paper, a comprehensive but brief review on smart grid communications technologies is presented. Section II describes smart grid communications technologies and their advantages and disadvantages. Section III mentions smart grid communications requirements in terms of security, system reliability, robustness, availability, scalability and the Quality of Service (QoS) mechanism. The standardization activities are reviewed in Section IV Finally, the paper is concluded in Section V.

\section{Communications Technologies AVAilable For SMART GRIDS}

A communications system is the key component of the smart grid infrastructure [1], [14], [16]. With the integration of advanced technologies and applications for achieving a smarter electricity grid infrastructure, a huge amount of data from different applications will be generated for further analysis, control and real-time pricing methods. Hence, it is very critical for electric utilities to define the communications requirements and find the best communications infrastructure to handle the output data and deliver a reliable, secure and cost effective service throughout the total system. Electric utilities attempt to get customer's attention to participate in the smart grid system, in order to improve services and efficiency. Demand side management and customer participation for efficient electricity usage are well understood, furthermore, the outages after disasters in existing power structure also focus the attention on the importance of the relationship between electric grids and communications systems [1].

Different communications technologies supported by two main communications media, i.e., wired and wireless, can be used for data transmission between smart meters and electric utilities. In some instances, wireless communications have some advantages over wired technologies, such as low cost infrastructure and ease of connection to difficult or unreachable areas. However, the nature of the transmission path may cause the signal to attenuate. On the other hand, wired solutions do not have interference problems and their functions are not dependent on batteries, as wireless solutions do.

Basically, two types of information infrastructure are needed for information flow in a smart grid system. The first flow is from sensor and electrical appliances to smart meters, the second is between smart meters and the utility's data centers. As suggested in [17], the first data flow can be accomplished through power line communication or wireless communications, such as ZigBee, 6LowPAN, Z-wave and others. For the second information flow, cellular technologies or the Internet can be used. Nevertheless, there are key limiting factors that should be taken into account in the smart metering deployment process, such as time of deployment, operational costs, the availability of the technology and rural/urban or indoor/outdoor environment, etc. The technology choice that fits one environment may not be suitable for the other. In the following, some of the smart grid communications technologies along with their advantages and disadvantages are briefly explained. An overview of smart grid communication technologies can be found in Table I

\section{A. ZigBee}

ZigBee is a wireless communications technology that is relatively low in power usage, data rate, complexity and cost of deployment. It is an ideal technology for smart lightning, energy monitoring, home automation, and automatic meter reading, etc. ZigBee and ZigBee Smart Energy Profile (SEP) have been realized as the most suitable communication standards for smart grid residential network domain by the U.S National Institute for Standards and Technology (NIST) [18]. The communication between smart meters, as well as among intelligent home appliances and in home displays, is very important. Many AMI vendors, such as Itron, Elster, and Landis Gyr, prefer smart meters, that the ZigBee protocol can be integrated into [37]. ZigBee integrated smart meters can communicate with the ZigBee integrated devices and control them. ZigBee SEP provides utilities to send messages to the 
Table I

SMART GRID COMMUNICATIONS TECHNOLOGIES

\begin{tabular}{|c|c|c|c|c|c|}
\hline Technology & Spectrum & Data Rate & Coverage Range & Applications & Limitations \\
\hline GSM & $\begin{array}{ll}900-1800 \\
\mathrm{MHz}\end{array}$ & $\begin{array}{l}\mathrm{Up} \text { to } 14.4 \\
\mathrm{Kpbs}\end{array}$ & $1-10 \mathrm{~km}$ & $\begin{array}{l}\text { AMI, Demand } \\
\text { Response, HAN }\end{array}$ & Low date rates \\
\hline GPRS & $\begin{array}{l}900-1800 \\
\mathrm{MHz}\end{array}$ & Up to $170 \mathrm{kbps}$ & $1-10 \mathrm{~km}$ & $\begin{array}{l}\text { AMI, Demand } \\
\text { Response, HAN }\end{array}$ & Low data rates \\
\hline 3G & $\begin{array}{l}1.92-1.98 \mathrm{GHz} \\
2.11-2.17 \mathrm{GHz} \\
\text { (licensed) }\end{array}$ & $\begin{array}{ll}384 & \text { Kbps-2 } \\
\text { Mbps } & \end{array}$ & $1-10 \mathrm{~km}$ & $\begin{array}{l}\text { AMI, Demand } \\
\text { Response, HAN }\end{array}$ & Costly spectrum fees \\
\hline WiMAX & $\begin{array}{l}2.5 \quad \mathrm{GHz}, \quad 3.5 \\
\mathrm{GHz}, 5.8 \mathrm{GHz}\end{array}$ & Up to $75 \mathrm{Mbps}$ & $\begin{array}{l}10-50 \mathrm{~km}(\mathrm{LOS}) \\
1-5 \mathrm{~km} \text { (NLOS) }\end{array}$ & $\begin{array}{ll}\text { AMI, } & \text { Demand } \\
\text { Response } & \end{array}$ & Not widespread \\
\hline PLC & 1-30 MHz & 2-3 Mbps & $1-3 \mathrm{~km}$ & AMI, Fraud Detection & $\begin{array}{l}\text { Harsh, noisy channel } \\
\text { environment }\end{array}$ \\
\hline ZigBee & $\begin{array}{l}2.4 \mathrm{GHz}-868- \\
915 \mathrm{MHz}\end{array}$ & $250 \mathrm{Kbps}$ & $30-50 \mathrm{~m}$ & AMI, HAN & $\begin{array}{l}\text { Low data rate, short } \\
\text { range }\end{array}$ \\
\hline
\end{tabular}

home owners, and home owners can reach the information of their real-time energy consumption.

1) Advantages: ZigBee has 16 channels in the $2.4 \mathrm{GHz}$ band, each with $5 \mathrm{MHz}$ of bandwidth. $0 \mathrm{dBm}(1 \mathrm{~mW})$ is the maximum output power of the radios with a transmission range between 1 and $100 \mathrm{~m}$ with a $250 \mathrm{~Kb} / \mathrm{s}$ data rate and OQPSK modulation [18]. ZigBee is considered as a good option for metering and energy management and ideal for smart grid implementations along with its simplicity, mobility, robustness, low bandwidth requirements, low cost of deployment, its operation within an unlicensed spectrum, easy network implementation, being a standardized protocol based on the IEEE 802.15.4 standard [4]. ZigBee SEP also has some advantages for gas, water and electricity utilities, such as load control and reduction, demand response, real-time pricing programs, real-time system monitoring and advanced metering support [18], [19].

2) Disadvantages: There are some constraints on ZigBee for practical implementations, such as low processing capabilities, small memory size, small delay requirements and being subject to interference with other appliances, which share the same transmission medium, license-free industrial, scientific and medical (ISM) frequency band ranging from IEEE 802.11 wireless local area networks (WLANs), WiFi, Bluetooth and Microwave [18]. Hence, these concerns about the robustness of ZigBee under noise conditions increase the possibility of corrupting the entire communications channel due to the interference of $802.11 / \mathrm{b} / \mathrm{g}$ in the vicinity of ZigBee [20]. Interference detection schemes, interference avoidance schemes and energy-efficient routing protocols, should be implemented to extend the network life time and provide a reliable and energy-efficient network performance.

\section{B. Wireless Mesh}

A mesh network is a flexible network consisting of a group of nodes, where new nodes can join the group and each node can act as an independent router. The self-healing characteristic of the network enables the communication signals to find another route via the active nodes, if any node should drop out of the network. Especially, in North America, RF mesh based systems are very popular. In PG\&E's SmartMeter system, every smart device is equipped with a radio module and each of them routes the metering data through nearby meters. Each meter acts as a signal repeater until the collected data reaches the electric network access point. Then, collected data is transferred to the utility via a communication network. A private company, SkyPilot Networks uses mesh networking for smart grid applications due to the redundancy and high availability features of mesh technology [37].

1) Advantages: Mesh networking is a cost effective solution with dynamic self-organization, self-healing, selfconfiguration, high scalability services, which provide many advantages, such as improving the network performance, balancing the load on the network, extending the network coverage range [21]. Good coverage can be provided in urban and suburban areas with the ability of multi-hop routing. Also, the nature of a mesh network allows meters to act as signal repeaters and adding more repeaters to the network can extend the coverage and capacity of the network. Advanced metering infrastructures and home energy management are some of the applications that wireless mesh technology can be used for.

2) Disadvantages: Network capacity, fading and interference can be counted as the major challenges of wireless mesh networking systems. In urban areas, mesh networks have been faced with a coverage challenge since the meter density cannot provide complete coverage of the communications network. Providing the balance between reliable and flexible routing, a sufficient number of smart nodes, taking into account node cost, are very critical for mesh networks. Furthermore, a third party company is required to manage the network, and since the metering information passes through every access point, some encryption techniques are applied to the data for security purposes. In addition, while data packets travel around many neighbors, there can be loop problems causing additional overheads in the communications channel that would result in a reduction of the available bandwidth [20].

\section{Cellular Network Communication}

Existing cellular networks can be a good option for communicating between smart meters and the utility and between far nodes. The existing communications infrastructure avoids utilities from spending operational costs and additional time for building a dedicated communications infrastructure. Cellular network solutions also enable smart metering deployments spreading to a wide area environment. $2 \mathrm{G}, 2.5 \mathrm{G}, 3 \mathrm{G}$, WiMAX and LTE are the cellular communication technologies 
available to utilities for smart metering deployments. When a data transfer interval between the meter and the utility of typically 15 minutes is used, a huge amount of data will be generated and a high data rate connection would be required to transfer the data to the utility. For example, T-Mobile's Global System for Mobile Communications (GSM) network is chosen for the deployment of Echelon's Networked Energy Services (NES) system. An embedded T-Mobile SIM within a cellular radio module will be integrated into Echelon's smart meters to enable the communication between the smart meters and the back-haul utility. Since T-Mobile's GSM network will handle all the communication requirements of the smart metering network, there is no need for an investment of a new dedicated communications network by utilities. Telenor, Telecom Italia, China Mobile, Vodafone have also agreed to put their GSM network into service for data flow of smart metering communications. Itron's SENITEL electricity meter is integrated with a GPRS module and communicates with a server running SmartSynch's Transaction Management System. CDMA, WCDMA and UMTS wireless technologies are also used in smart grid projects. A CDMA smart grid solution for the residential utility market has been introduced by Verizon, and Verizon's 3G CDMA network will be used as the backbone of the smart grid communications with the SmartSynch smart grid solutions [37]. UMTS is IP-based and a packet oriented service that is suitable for metering applications [37]. Telenor with Cinclus technology is offering UMTS technology for smart grid communications [37].

An Australian energy delivery company, SP AusNet, is building a dedicated communications network for smart grid applications and chose WiMAX technology for the communications need of the smart meters. WiMAX chip sets are embedded into the smart meters and wireless communications is dedicated between smart meters and the central system in SP AusNet's system. A U.S. wireless carrier, Sprint Nextel, had signed a partnership with the smart grid software provider, Grid Net, on a project to provide communication between smart meters and smart routers over its $4 \mathrm{G}$ wireless network. General Electric (GE) is developing WiMAX based smart meters with CenterPoint Energy and had collaborated with Grid Net, Motorola and Intel to focus on WiMAX connectivity solutions. In GE's smart meter project with CenterPoint Energy, it will deploy WiMAX based MDS Mercury 3650 radios to connect the utility's back-haul system to collection points, which will collect data from smart meters that are installed by CenterPoint [37]. Furthermore, some major companies, such as Cisco, Silver Springs Network and Verizon, also implement WiMAX smart grid applications. The world's largest WiMAX vendor, Alvarion, has announced its partnership with a U.S. utility company, National Grid, for a WiMAX based smart grid project. Lower deployment and operating costs, proper security protocols, smooth communications, high data speeds (up to $75 \mathrm{Mbps}$ ), an appropriate amount of bandwidth and scalability are the advantages of today's WiMAX technology.

1) Advantages: Cellular networks already exist. Therefore, utilities do not have to incur extra cost for building the communications infrastructure required for a smart grid. Wide-spread and cost-effective benefits make cellular communication one of the leading communications technologies in the market. Due to data gathering at smaller intervals, a huge amount of data will be generated and the cellular networks will provide sufficient bandwidth for such applications. When security comes into discussion, cellular networks are ready to secure the data transmissions with strong security controls. To manage healthy communications with smart meters in rural or urban areas, the wide area deployment capability of smart grid becomes a key component and since the cellular networks coverage has reached almost $100 \%$. In addition, GSM technology performs up to $14.4 \mathrm{Kbps}$, GPRS performs up to $170 \mathrm{Kbps}$ and they both support AMI, Demand Response, Home Area Network (HAN) applications. Anonymity, authentication, signaling protection and user data protection security services are the security strengths of GSM technology [37]. Lower cost, better coverage, lower maintenance costs and fast installation features highlight why cellular networks can be the best candidate as a smart grid communications technology for the applications, such as demand response management, advanced metering infrastructures, HAN, outage management, etc.

2) Disadvantages: Some power grid mission-critical applications need continuous availability of communications. However, the services of cellular networks are shared by customer market and this may result in network congestion or decrease in network performance in emergency situations. Hence, these considerations can drive utilities to build their own private communications network. In abnormal situations, such as a wind storm, cellular network providers may not provide guarantee service. Compared to public networks, private networks may handle these kinds of situations better due to the usage of a variety of technologies and spectrum bands.

\section{Power Line Communication}

Power line communication (PLC) is a technique that uses the existing power lines to transmit high speed ( $2-3 \mathrm{Mbps})$ data signals from one device to the other. PLC has been the first choice for communication with the electricity meter due to the direct connection with the meter [20] and successful implementations of AMI in urban areas where other solutions struggle to meet the needs of utilities. PLC systems based on the LV distribution network have been one of the research topics for smart grid applications in China [22]. In a typical PLC network, smart meters are connected to the data concentrator through power lines and data is transferred to the data center via cellular network technologies. For example, any electrical device, such as a power line smart transceiver-based meter, can be connected to the power line and used to transmit the metering data to a central location [37]. France has launched the "Linky meter project" that includes updating 35 million traditional meters to Linky smart meters. PLC technology is chosen for data communication between the smart meters and the data concentrator, while GPRS technology is used for transferring the data from the data concentrator to the utility's data center [37]. ENEL, the Italian electric utility, chose PLC technology to transfer smart meter data to the nearest data concentrator and GSM technology to send the data to data centers. 
1) Advantages: PLC can be considered as a promising technology for smart grid applications due to the fact that the existing infrastructure decreases the installation cost of the communications infrastructure. The standardization efforts on PLC networks, the cost-effective, ubiquitous nature and widely available infrastructure of PLC, can be the reasons for its strength and popularity [23]. Data transmissions are broadcast in nature for PLC, hence, the security aspects are critical. Confidentiality, authentication, integrity and user intervention are some of the critical issues in smart grid communications. HAN application is one of the biggest applications for PLC technology. Moreover, PLC technology can be well suited to urban areas for smart grid applications, such as smart metering, monitoring and control applications, since the PLC infrastructure is already covering the areas that are in the range of the service territory of utility companies.

2) Disadvantages: There are some technical challenges due to the nature of the power line networks. The power line transmission medium is a harsh and noisy environment that makes the channel difficult to be modeled. The lowbandwidth characteristic (20 kbps for neighborhood area networks) restricts the PLC technology for applications that need higher bandwidth [37]. Furthermore, the network topology, the number and type of the devices connected to the power lines, wiring distance between transmitter and receiver, all, adversely affect the quality of signal, that is transmitted over the power lines [37]. The sensitivity of PLC to disturbances and dependency on the quality of signal are the disadvantages that make PLC technology not suited for data transmission. However, there have been some hybrid solutions in which PLC technology is combined with other technologies, i.e., GPRS or GSM, to provide full-connectivity not possible by PLC technology.

\section{E. Digital Subscriber Lines}

Digital Subscriber Lines (DSL) is a high speed digital data transmission technology that uses the wires of the voice telephone network. It is common to see frequencies greater than $1 \mathrm{MHz}$ through an ADSL enabled telephone line [16]. The already existing infrastructure of DSL lines reduces installation cost. Hence, many companies chose DSL technology for their smart grid projects. The Current Group, a Smart Grid Solution Company, has collaborated with Qwest to implement a Smart Grid project. Qwest's existing low latency, secure, high capacity DSL network will be used for data transmissions. Xcel Energy's "SmartGridCity" project has also proved the interoperability of the technology by utilizing the Current's intelligent sensors and OpenGrid platform and Qwest's DSL network. A smart metering project has been carried out for Stadtwerke Emden-Municipal Utilities in Germany by Deutsche Telekom. In the project, Deutsche Telekom is responsible to provide the data communications for electric and gas meters. A communication box will be installed at the customer premises and the consumption information will be transmitted over DSL to Stadtwerke Emden [37]. Deutsche Telekom offers many services in this project, such as reading consumption data, installation and operation, data transmission, etc. However, the throughput of the DSL connection depends on how far away the subscriber is from the serving telephone exchange and this makes it difficult to characterize the performance of DSL technology [16].

1) Advantages: The widespread availability, low cost and high bandwidth data transmissions are the most important reasons for making the DSL technology the first communications candidate for electricity suppliers in implementing the smart grid concept with smart metering and data transmission smart grid applications.

2) Disadvantages : The reliability and potential down time of DSL technology may not be acceptable for mission critical applications. Distance dependence and lack of standardization may cause additional problems. The wired DSL-based communications systems require communications cables to be installed and regularly maintained, and thus, cannot be implemented in rural areas due to the high cost of installing fixed infrastructure for low-density areas.

To conclude, wired technologies, such as DSL, PLC, optical fiber, are costly for wide area deployments but they have the ability to increase the communications capacity, reliability and security. On the other hand, wireless technologies can reduce the installation costs, but provide constrained bandwidth and security options.

\section{SMART GRID COMmunications Requirements}

The communication infrastructure between energy generation, transmission, and distribution and consumption requires two-way communications, inter-operability between advanced applications and end-to-end reliable and secure communications with low-latencies and sufficient bandwidth [25]; Moreover, the system security should be robust enough to prevent cyber-attacks and provide system stability and reliability with advanced controls. In the following, major smart grid communication requirements are presented.

\section{A. Security}

Secure information storage and transportation are extremely vital for power utilities, especially for billing purposes and grid control [24]. To avoid cyber-attacks, efficient security mechanisms should be developed and standardization efforts regarding the security of the power grid should be made.

\section{B. System Reliability, Robustness and Availability}

Providing the system reliability has become one of the most prioritized requirements for power utilities. Aging power infrastructure and increasing energy consumption and peak demand are some of the reasons that create unreliability issues for the power grid [26]. Harnessing the modern and secure communication protocols, the communication and information technologies, faster and more robust control devices, embedded intelligent devices (IEDs) for the entire grid from substation and feeder to customer resources, will significantly strengthen the system reliability and robustness [26]. The availability of the communication structure is based on preferred communication technology. Wireless technologies with constrained bandwidth and security and reduced installation 
costs can be a good choice for large-scale smart grid deployments [24]. On the other hand, wired technologies with increased capacity, reliability and security can be costly [24]. To provide system reliability, robustness and availability at the same time with appropriate installation costs, a hybrid communication technology mixed with wired and wireless solutions can be used.

\section{Scalability}

A smart grid should be scalable enough to facilitate the operation of the power grid [3]. Many smart meters, smart sensor nodes, smart data collectors, and renewable energy resources are joining the communications network. Hence, smart grid should handle the scalability with the integration of advanced web services, reliable protocols with advanced functionalities, such as self-configuration, security aspects.

\section{Quality of Service (QoS)}

The communication between the power supplier and power customers is a key issue of the smart grid. Performance degradation like delay or outage may compromise stability, therefore, a QoS mechanism must be provided to satisfy the communications requirements (for example high speed routing) and a QoS routing protocol must be applied in the communications network. This incurs two important questions unique to smart grid:

- How to define the QoS requirement in the context of smart grid.

- How to ensure the QoS requirement from the home appliance in the communications network.

To answer the first question, the detailed mechanism of power price, based on the dynamics of the load, must be investigated. Then, a reward system is built for the home appliance based on the power price and the utility function of the appliance, thus obtaining the impact of delay and outage on the reward of the home appliance. Finally, the QoS requirement is derived by optimizing the reward [27]. To answer the second question, routing methodologies meeting the derived QoS requirement are focused on. Due to the requirements of high computing and storage capabilities imposed by the heterogeneity of the smart grid, multiple QoS-aware routing within multiple (more than 2) constraints must be considered (for example a greedy algorithm with $\mathrm{K}$-approximation, where $\mathrm{K}$ is the number of constraints) [27]. A QoS requirement usually includes specifications, like average delay, jitter and connection outage probability. To derive the QoS requirement, it is important to describe the probabilistic dynamics of the power system, to evaluate the impact of different QoS specifications on the smart grid system and to derive the QoS requirement from the corresponding impact.

The power price is typically determined by locational margin price (LMP) [28] driven by the load that varies with time. A constrained optimization problem can be used to derive the LMP from the load and other parameters, where the Lagrange factors of the constraints are considered as prices.

To efficiently link together the large number of smart grid components, a powerful data communications infrastructure will be provided. It is expected that part of this infrastructure will make use of the power distribution lines themselves as communications carriers using PLC technology [29]. It is also expected to have a combination of wireless technologies to establish a reliable communications infrastructure. Also, recent standardization efforts under the umbrella of IEEE (P1901.2), ITU (ITU-G.hnem), and others are dedicated to PLC technology for Smart Grid applications.

One of the challenges of employing PLC in power distribution grids is multi-hop transmission message routing. The basic idea is that network nodes, i.e., PLC enabled devices, act as repeaters of messages in order to achieve sufficient coverage [30], [31]. The focus in these two previous studies is on reliable delivery of messages taking into account unpredictable and possible sudden changes of communications links and network topology. In this regard, for flooding of messages, the concept of single-frequency network (SFN) transmission is presented in [30], [31]. In [32], the problem of routing in PLC networks is revised taking into account that network nodes are static and thus, their location is known a priori. In other words, the nodes know in which direction a message is intended to flow. More specifically, if a node receives a packet it can decide whether to forward it or not. Such routing algorithms are known as geographic routing in the wireless communications literature [33], where they have been applied mainly in the context of wireless sensor networks. These algorithms present high performance for the application at hand: they close the gap between flooding on the one hand and improved shortest path routing on the other.

In [34], the implementation of a smart monitoring system over a wireless sensor network is presented, with particular emphasis on the creation of a solid routing infrastructure through the routing protocol for low-power and lossy networks (RPL), whose definition is currently being discussed within the IETF ROLL working group. RPL was designed in order to match the requirements of networks characterized by low power supplies and by deployment in lossy environments. This involves both wired and wireless networks deployed in difficult environments, where the presence of high interference requires adaptive and reconfigurable network operations. In [35] a hybrid routing protocol that combines local agility with centralized control is presented. It meets the requirements of robust collection, point-to-point communication, and low footprint. It uses a distributed algorithm to form a Directed Acyclic Graph (DAG) for routing data from in-network nodes to border routers, allowing nodes to maintain multiple options that are ranked through data-driven link estimation.

\section{SMART GRID STANDARDS}

There are many applications, techniques and technological solutions for smart grid system that have been developed or are still in the development phase. However, the key challenge is that the overall smart grid system is lacking widely accepted standards and this situation prevents the integration of advanced applications, smart meters, smart devices and renewable energy sources and limits the inter-operability between them. The adoption of inter-operability standards 
for the overall system is a critical prerequisite for making the smart grid system a reality. Seamless interoperability, robust information security, increased safety of new products and systems, compact set of protocols and communication exchange are some of the objectives that can be achieved with smart grid standardization efforts [37]. There are many regional and national attempts towards achieving this goal; for example, the European Union Technology Platform organization's strategic energy technology plan is all about the development of a smart electricity system over the next 30 years; Ontario Energy Board, Canada, has committed itself towards the completion of a smart meter installation [37]. On the other hand, NIST, the American National Standards Institute (ANSI), the International Electro technical Commission (IEC), the Institute of Electrical and Electronics Engineers (IEEE), the International Organization for Standardization (ISO), the International Telecommunication Union (ITU), the 3rd Generation Partnership Project (3GPP) and on the regional level, the Korean Agency for Technology and Standards (KATS) and Joint Inannounced formation Systems Committee(JISC) are the recognized standard development organizations that are worth to mention. In addition, the CEN, CENELEC and ETSI has formed a Joint working group for smart grid standardization efforts and aim to achieve the European Commission's policy objectives regarding the smart grid [37]. Their efforts focus on smart metering functionalities and communication interfaces for electric, water and heat sectors in Europe. An overview of smart grid standards are given in Table III In the following, the details of these standards are explained.

\section{A. Revenue Metering Information Model}

- ANSI C12.19: ANSI C12.19 is an ANSI standard for utility industry end device data tables. This standard is defining a table structure for data transmissions between an end device and a computer for utility applications using binary codes and XML content. ANSI C12.19 is not interested in defining device design criteria or specifying the language or protocol used to transport that data.

- M-Bus: M-Bus is a European standard and provides the requirements for remotely reading all kinds of utility meters. The utility meters are connected to a common master that periodically reads the meters via M-Bus. The wireless version, Wireless M-Bus, is also specified recently.

- ANSI C12.18: ANSI C12.18 is an American National Standard (ANSI) standard that is specifically designed for meter communications and responsible for two way communications between smart electricity meters $(\mathrm{C} 12.18$ device) and a C12.18 client via an optical port.

\section{B. Building Automation}

- BACnet: BACnet is a standard communication protocol that was developed by the American Society of Heating, Refrigerating and Air-Conditioning Engineers (ASHRAE) for building automation and control networks and support the implementation of intelligent buildings with full integration of computer-based building automation and control systems from multiple manufacturers.

\section{Substation Automation}

- IEC 61850: IEC 61850 is a flexible, open standard that defines the communication between devices in transmission, distribution and substation automation systems. To enable seamless data communications and information exchange between the overall distribution networks, it is aimed to increase the scope of IEC 6180 to whole electric network and provide its compatibility with Common Information Model (CIM) for monitoring, control and protection applications [25]. This technology is implemented by modern manufacturers in their latest power engineering products like distribution automation nodes/grid measurement and diagnostics devices [13].

\section{Powerline Networking}

- HomePlug: HomePlug is a power line technology and the existing home electricity is used to connect the smart appliances to HAN; HomePlug Command and Control (HPCC) version is designed for low-cost applications. HomePlug is a promising technology to create a reliable HAN between electric appliances and a smart meter.

- HomePlug Green PHY: HomePlug Green PHY specification is developed as a low power, cost-optimized power line networking specification standard for smart grid applications used in home area networking by the Smart Energy Technical Working Group within the HomePlug Powerline Alliance. The inputs for optimization of specifications for field tests were gathered from many utilities, i.e., Consumers Energy, Duke Energy, Pacific Gas and Electric, and Southern California Edison. Backwards interoperability, lower data rate and IP networking support, low power consumption, full interoperability with both HomePlug devices are the leading features of HomePlug Green PHY specification.

- PRIME: PRIME is an open, global power line standard that provides multi-vendor interoperability and welcomes several entities to its body. Advanced Digital Design, CURRENT Group, Landis+Gyr, STMicroelectronics, uSyscom and ZIV Medida are some of the current companies that have extensive experience in PLC technology and smart metering.

- G3-PLC: G3-PLC is a power line communications specification launched by ERDF and Maxim that aims to provide interoperability, cyber security and robustness and reduce infrastructure costs in smart grid implementations worldwide.

\section{E. Home Area Network Device Communication Measurement} and Control

- U-SNAP: There have been a variety of incompatible standards for HAN. This lack of standardization in HAN Utility has driven major AMI suppliers and product manufacturers to develop a solution, namely Utility Smart 
Table II

OVERVIEW OF SMART GRID STANDARDS

\begin{tabular}{|c|c|c|}
\hline $\begin{array}{l}\text { Type/Name of } \\
\text { Standards }\end{array}$ & Details & Application \\
\hline IEC 61970 & Providing Common Information Model (CIM): IEC & Energy management systems \\
\hline $\begin{array}{l}\text { and } \\
61969\end{array}$ & $\begin{array}{l}61970 \text { works in the transmission domain and IEC } \\
61969 \text { works in the distribution domain }\end{array}$ & \\
\hline IEC61850 & $\begin{array}{l}\text { Flexible, future proofing, open standard, communica- } \\
\text { tion between devices in transmission, distribution and } \\
\text { substation automation systems }\end{array}$ & Substation Automation \\
\hline $\begin{array}{l}\text { IEC } 60870-6 \\
\text { /TASE.2 }\end{array}$ & $\begin{array}{l}\text { Data exchange between utility control centers, utilities, } \\
\text { power pools, regional control centers }\end{array}$ & Inter-control center communications \\
\hline $\begin{array}{l}\text { IEC } 62351 \\
\text { Parts } 1-8\end{array}$ & $\begin{array}{l}\text { Defining cyber security for the communication proto- } \\
\text { cols }\end{array}$ & Information Security Systems \\
\hline IEEE P2030 & $\begin{array}{l}\text { A Guide for smart grid inter-operability of energy } \\
\text { technology and IT operation with the electric power } \\
\text { system (EPS) }\end{array}$ & Customer-side applications \\
\hline IEEE P1901 & High speed power line communications & $\begin{array}{l}\text { In-home multimedia, utility and smart grid } \\
\text { applications }\end{array}$ \\
\hline $\begin{array}{l}\text { ITU-T G.9955 } \\
\text { and G.9956 }\end{array}$ & $\begin{array}{l}\text { ITU-T G.9955 and G.9956 contain the physical layer } \\
\text { specification and the data link layer specification }\end{array}$ & Distribution Automation, AMI \\
\hline OpenADR & Dynamic pricing, Demand Response & Price Responsive and Load Control \\
\hline BÄCnet & Scalable system communications at customer side & Building automation \\
\hline HomePlug & $\begin{array}{l}\text { Powerline technology to connect the smart appliances } \\
\text { to HAN }\end{array}$ & HAN \\
\hline $\begin{array}{l}\text { HomePlug } \\
\text { Green PHY }\end{array}$ & $\begin{array}{l}\text { Specification developed as a low power, cost-optimized } \\
\text { power line networking specification standard for smart } \\
\text { grid applications }\end{array}$ & HAN \\
\hline U-SNAP & $\begin{array}{l}\text { Providing many communication protocols to connect } \\
\text { HAN devices to smart meters }\end{array}$ & HAN \\
\hline ISA100.11a & Open standard for wireless systems & Industrial Automation \\
\hline SAE J2293 & $\begin{array}{l}\text { Standard for the electrical energy transfer from electric } \\
\text { utility to EVs }\end{array}$ & Electric Vehicle Supply Equipment \\
\hline ANSI C12.22 & $\begin{array}{l}\text { Data network communications are supported and } \\
\text { C12.19 tables are transported }\end{array}$ & AMI \\
\hline ANSI C12.18 & $\begin{array}{l}\text { Data structures transportation via the infrared optical } \\
\text { port han }\end{array}$ & AMI \\
\hline ANSI C12.19 & $\begin{array}{l}\text { Flexible metering model for common data structures } \\
\text { and industry "vocabulary" for meter data communica- } \\
\text { tions }\end{array}$ & AMI \\
\hline Z-Wave & $\begin{array}{l}\text { Alternative solution to ZigBee that handles the inter- } \\
\text { ference with } 802.11 / \mathrm{b} / \mathrm{g}\end{array}$ & HAN \\
\hline M-Bus & $\begin{array}{l}\text { European standard and providing the requirements for } \\
\text { remotely reading all kinds of utility meters }\end{array}$ & AMI \\
\hline PRIME & Open, global standard for multi-vendor interoperability & AMI \\
\hline G3-PLC & $\begin{array}{l}\text { Providing interoperability, cyber security, and robust- } \\
\text { ness }\end{array}$ & AMI \\
\hline SAE J2836 & $\begin{array}{l}\text { Supporting use cases for plug-in electric vehicles com- } \\
\text { munication }\end{array}$ & Electric Vehicle \\
\hline SAE J2847 & $\begin{array}{l}\text { Supports communication messages between PEVs and } \\
\text { grid components }\end{array}$ & Electric Vehicle \\
\hline
\end{tabular}

Network Access Port (U-SNAP). The main requirement is the existence of an interface to connect any type of product to a HAN. U-SNAP basically enables the standardization of a connector and serial interface and identifies the hardware interface, physical dimensions, data transfer, message contents and protocol specifics for HAN devices to provide many communication protocols to connect HAN devices to smart meters.

- IEEE P1901: The IEEE P1901 Working Group (WG) under the sponsorship of the IEEE Communications Society developed the IEEE P1901 standard for high speed power line communications to meet in-home multimedia, utility and smart grid application requirements [36]. Access control and physical layer specifications for broadband over power line networks are analyzed in detail and the access system with cell structure is defined by the IEEE
P1901Working Group [36]. The IEEE P1901 standard has an important effect on communications technology by integrating power line communications into wireless networks with extensive features, such as high-speed, walls-penetration, etc.

- Z-Wave: Z-Wave is an alternative solution to ZigBee that handles the interference with $802.11 / \mathrm{b} / \mathrm{g}$ since it operates in the $800 \mathrm{MHz}$ range [20]. Z-Wave is not an open standard and developed by The Z-Wave Alliance, an international consortium of manufacturers. The simple, modular and low-cost features make Z-Wave one of the leading wireless technologies in home automation. ZWave can be easily embedded to consumer electronic appliances, such as lighting, remote control, security systems that require low-bandwidth data operations. 


\section{F. Application-Level Energy Management Systems}

- IEC 61970 and IEC 61968: Two defined standards, i.e., IEC 61970 and IEC 61969, provide what is called a Common Information Model (CIM), which is necessary for exchanging data between devices and networks. IEC 61970 works in the transmission domain, while IEC 61968 works in the distribution domain. CIM standards are integral to the deployment of a smart grid scenario, in which many devices connect to a single network.

- OpenADR : OpenADR is a research and standards development effort that is defined as a fully-automated demand response using open standard, platform-independent and transparent end-to-end technologies or software systems. OpenADR was originally developed at Lawrence Berkeley Labs, a U.S. government research laboratory. The adoption of OpenADR to the smart grid is very important to provide effective deployment of dynamic pricing, demand response and grid reliability.

\section{G. Inter-control and Inter-operability Center Communications}

- IEEE P2030: IEEE P2030 is a guide for smart grid interoperability of energy technology and information technology operation with the electric power system (EPS), and customer-side applications. Basically, IEEE P2030 can be understood as a single document for the inter-operability of smart grid. IEEE P2030 is responsible for seamless data transfer in a two-way communications manner for electric generation, reliable power delivery and customerside applications. The IEEE Standards Board, as sponsored by the IEEE Standards Coordinating Committee, confirmed the development of the IEEE P2030 smart grid inter-operability standard. Understanding and defining smart grid inter-operability of the power system with customer applications was the initial goal of IEEE P2030. Integration of energy technology and ICT, seamless communication between electric generation, delivery, and end-use benefits and increasing knowledge about grid architecture to provide reliable, flexible and secure electric delivery are some of the benefits of IEEE P2030.

- ANSI C12.22: ANSI C12.22 is defining a protocol for transporting ANSI C12.19 table data over networks to achieve the inter-operability among communications modules and smart meters by using AES encryption for enabling strong, secure communications, including confidentiality and data integrity .

- ISA100.11a: ISA100.11a is an open standard for wireless systems for industrial automation developed by the International Society of Automation (ISA). The ISA100.11a focuses on robustness, security and network management requirements of wireless infrastructure and low power consuming devices that provides large-scale installations. The ISA100.11a standard is simple to use and deploy and provides multi-vendor device interoperability.

- ITU-T G.9955 and G.9956: The two G.hnem standards ITU-T G.9955 and G.9956 contain the physical layer specification and the data link layer specification, respectively, for narrowband OFDM power line com- munications transceivers for communications via alternating current and direct current electric power lines over frequencies below $500 \mathrm{kHz}$. These ITU-T standards support indoor and outdoor communications over low voltage lines, medium voltage lines, through transformer low-voltage to medium-voltage, and through transformer medium-voltage to low-voltage power lines in both urban and long distance rural communications.

\section{H. Cyber Security}

- IEC 62351: IEC 62351 defines cyber security for the communication protocols defined by the previous four sets. Security is a major concern with smart grids, which are especially vulnerable to attack because of the twoway communication between devices and the utility grid.

\section{Electric Vehicles}

- SAE J2293: This standard was developed by the Hybrid Committee that is a part of SAE international and provides requirements for Electric Vehicles (EV) and Electric Vehicle Supply Equipment (EVSE). It standardizes the electrical energy transfer from electric utility to EVs.

- SAE J2836: SAE J2836 standard supports use cases for communication between plug-in electric vehicles and the power grid for energy transfer and other applications.

- SAE J2847: SAE J2847 supports communication messages between PEVs and grid components.

\section{CONCLUSION}

The smart grid has been conceived as an evolution of electric power systems due to the increasing diffusion of distributed generation by renewable sources, but with the additional aim to enhance efficiency, reliability and safety of the existing power grid. To this end, remote and timely information gathering about equipment failures, capacity limitations, and natural accidents is extremely critical for ensuring proactive and real-time and reliable diagnosis of possible failures in the smart grid. This makes cost-effective remote sensing technologies vital for safe, seamless and efficient power delivery in the smart grid.

In this paper, communications technologies and requirements for smart grids have been discussed. The QoS mechanism is introduced and standards are presented. Clearly, there are many important open research issues for the realization of smart grid communications and applications. Future work includes discussion of grid characteristics, architectures, key players, pilot projects, applications and research challenges on ICT issues, in order to give a complete overview on the subject.

\section{ACKNOWLEDGEMENTS}

The work of V.C. Gungor, D. Sahin, T. Kocak, and S. Ergüt was funded by Türk Telekom under Award Number 11316-01 and the work of C. Buccella and C. Cecati was funded by DigiPower s.r.l., L'Aquila, Italy, and the work of G.P. Hancke was funded by Eskom, South Africa. 


\section{REFERENCES}

[1] V.C. Gungor, B. Lu, G.P. Hancke, "Opportunities and Challenges of Wireless Sensor Networks in Smart Grid," IEEE on Trans. Ind. Electron.,vol. 57, no. 10, pp. 3557-3564, October 2010. I] II

[2] U.S Department of Energy, 2011, Avaliable: http://www.oe.energy.gov. I]

[3] V.C. Gungor, G. Hancke, "Industrial Wireless Sensor Networks: Challenges, Design Principles, and Technical Approaches," IEEE Trans. on Industrial Electronics, vol. 56, no. 10, pp. 42584265, October 2009. III-C

[4] B. Lu, V.C. Gungor, "Online and Remote Energy Monitoring and Fault Diagnostics for Industrial Motor Systems using Wireless Sensor Networks," IEEE Trans. on Industrial Electronics, vol. 56, no. 11, November 2009. II-A1

[5] M. Erol-Kantarci, H.T. Mouftah, "Wireless multimedia sensor and actor networks for the next generation power grid" Ad Hoc Networks, vol. 9, no. 5, pp. 542-551, June 2011. I]

[6] A.Y. Saber, G.K. Venayagamoorthy, "Plug-in Vehicles and Renewable Energy Sources for Cost and Emission Reductions," IEEE Trans. on Industrial Electronics, vol.58, no.4, pp.12291238, April 2011. [I

[7] D. Lu, H. Kanchev, F. Colas, V. Lazarov, B. Francois, "Energy management and operational planning of a microgrid with a PV-based active generator for Smart Grid Applications," IEEE Trans. on Industrial Electronics, Digital Object Identifier: 10.1109/TIE.2011.2119451. I]

[8] P. Palensky, D. Dietrich, "Demand Side Management: Demand Response, Intelligent Energy Systems, and Smart Loads," IEEE Trans. on Industrial Informatics, Inf., vol. PP, No. 99, Digital Object Identifier: 10.1109/TII.2011.2158841 []

[9] V. Calderaro, C. Hadjicostis, A. Piccolo, P. Siano, "Failure Identification in Smart Grids based on Petri Net Modeling," IEEE Trans. on Industrial Electronics, Digital Object Identifier: 10.1109/TIE.2011.2109335. []

[10] C. Cecati, C. Citro, P. Siano, "Combined Operations of Renewable Energy Systems and Responsive Demand in a Smart Grid," IEEE Trans. on Sustainable Energy, in press. Digital Object Identifier: 10.1109/TSTE.2011.2161624. []

[11] P. Siano, C. Cecati, C. Citro, P. Siano, "Smart Operation of Wind Turbines and Diesel Generators According to Economic Criteria," IEEE Trans. on Industrial Electronics, vol. 58, no. 10, pp.— (in press) Digital Object Identifier: 10.1109/TIE.2011.2106100. []

[12] A. Vaccaro, G. Velotto, A. Zobaa, "A Decentralized and Cooperative Architecture for Optimal Voltage Regulation in Smart Grids," IEEE Trans. on Industrial Electronics,, Digital Object Identifier: 10.1109/TIE.2011.2143374. []

[13] D. Dietrich, D. Bruckner, G. Zucker, P. Palensky, , "Communication and Computation in Buildings: A Short Introduction and Overview," IEEE Trans. on Industrial Electronics,, vol.57, no.11, pp.3577-3584, Nov. 2010 IV-C

[14] V.C. Gungor, F. C. Lambert, "A Survey on Communication Networks for Electric System Automation," Computer Networks, vol. 50, pp. 877-897, May 2006. [1] [I]

[15] S. Paudyal, C. Canizares, K. Bhattacharya, "Optimal Operation of Distribution Feeders in Smart Grids," IEEE Trans. on Industrial Electronics, Digital Object Identifier: 10.1109/TIE.2011.2112314. []

[16] D. M. Laverty, D. J. Morrow, R. Best, P. A. Crossley, "Telecommunications for Smart Grid: Backhaul solutions for the distribution network," IEEE Power and Energy Society General Meeting, pp. 1-6, 25-29 July 2010. II II-E

[17] L. Wenpeng, D. Sharp, S. Lancashire, "Smart grid communication network capacity planning for power utilities," IEEE PES, Transmission and Distribution Conference and Exposition, pp. 1-4, 19-22 April 2010. II

[18] Y. Peizhong, A. Iwayemi, C. Zhou, "Developing ZigBee Deployment Guideline Under WiFi Interference for Smart Grid
Applications," IEEE Trans. on Smart Grid, vol.2, no.1, pp. 110120, March 2011. II-A II-A1 II-A2

[19] C. Gezer, C. Buratti,"A ZigBee Smart Energy Implementation for Energy Efficient Buildings," in Proc. of IEEE Vehicular Technology Conference (VTC Spring), 73rd, vol., no., pp.15, 15-18 May 2011 [II-A1

[20] R. P. Lewis, P. Igic and Z. Zhongfu, "Assessment of communication methods for smart electricity metering in the U.K.," in Proc. of IEEE PES/IAS Conference on Sustainable Alternative Energy (SAE), pp. 1-4, Sept. 2009. III-A2 II-B2 II-D IV-E

[21] A. Yarali, "Wireless Mesh Networking technology for commercial and industrial customers," in Proc. of Electrical and Computer Engineering, CCECE 2008. [II-B1]

[22] M.Y. Zhai, "Transmission Characteristics of Low-Voltage Distribution Networks in China Under the Smart Grids Environment," IEEE Trans. on Power Delivery, vol.26, no.1, pp. 173180, Jan. 2011. II-D

[23] V. Paruchuri, A. Durresi, M. Ramesh , "Securing powerline communications," IEEE Int. Symp. on Power Line Communications and Its Applications (ISPLC), pp. 64-69, 2-4 April 2008. II-D1

[24] Q. Yang, J. A. Barria, T.C. Green, "Communication Infrastructures for Distributed Control of Power Distribution Networks," IEEE Trans. on Industrial Informatics, vol.7, no.2, pp. 316-327, May 2011. [II-A III-B

[25] T. Sauter, M. Lobashov, "End-to-End Communication Architecture for Smart Grids," IEEE Trans. on Industrial Electronics, vol.58, no.4, pp. 1218-1228, April 2011. III IV-C

[26] K. Moslehi, R. Kumar, "Smart Grid - a reliability perspective," Innovative Smart Grid Technologies (ISGT), pp.1-8, 19-21 Jan. 2010. III-B

[27] Southern Company Services, Inc., Comments Request for Information on Smart Grid Communications Requirements, July 2010. Available: http://www.alvarion.com/index.php/. III-D

[28] R. Bo, F. Li, "Probabilistic LMP forecasting considering load uncertainty," IEEE Trans. on Power Systems, vol. 24, pp. 1279-1289, Aug. 2009. III-D

[29] H. Ferreira, L. Lampe, J. Newbury, T. Swart (Editors), Power Line Communications, John Wiley \& Sons, 2010. III-D

[30] G. Bumiller, "Single Frequency Network Technology for Fast ad hoc Communication Networks over Power Lines," WiKuWissenschaftsverlag Dr. Stein, 2010. III-D

[31] G. Bumiller, L. Lampe, H. Hrasnica, "Power Line Communications for Large-Scale Control and Automation Systems," IEEE Communications Magazine, vol. 48, no. 4, pp. 106-113, Apr. 2010. IIII-D

[32] M. Biagi, L. Lampe, "Location Assisted Routing Techniques for Power Line Communication in Smart Grids," in Proc. of IEEE Int. Conf. on Smart Grid Communications, pp. 274 - 278 , 2010. ПII-D

[33] J. Sanchez, P. Ruiz, R. Marin-Perez, "Beacon-less geographic routing made partical: Challenges, design guidelines and protocols," IEEE Communications Magazine, vol. 47, no. 8, pp. 85-91, Aug. 2009. III-D

[34] N. Bressan, L. Bazzaco, N. Bui, P. Casari, L. Vangelista, M Zorzi, "The Deployment of a Smart Monitoring System using Wireless Sensors and Actuators Networks," in Proc. of IEEE Int. Conf. on Smart Grid Communications (SmartGridComm), pp. 49 - 54, 2010. III-D

[35] S. Dawson-Haggerty, A. Tavakoli and D. Culler, "Hydro: A Hybrid Routing Protocol for Low-Power and Lossy Networks," in Proc. of IEEE International Conference on Smart Grid Communications (SmartGridComm), pp. 268 - 273, 2010. III-D

[36] S. Goldfisher and S.J. Tanabe, "IEEE 1901 access system: An overview of its uniqueness and motivation," IEEE Communications Magazine, vol. 48, no. 10, pp. 150-157, October 2010. IV-E

[37] V.C. Gungor, D. Sahin, T. Kocak, and S. Ergüt, 'Smart Grid Communications and Networking," Türk Telekom Technical Report-11316-01, April, 2011. 